INRA Prod. Anim., 2007, 20 (2), 129-136

\title{
Prévision de la digestibilité des fourrages par la méthode pepsine-cellulase. Le point sur les équations proposées
}

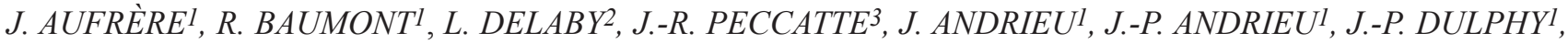 \\ ${ }^{1}$ INRA, UR1213 Herbivores, F-63122 Saint-Genès Champanelle, France \\ 2 INRA, Agrocampus, UMR1080 Production du lait, F-35590 Saint-Gilles, France \\ ${ }^{3}$ INRA , UE326 Domaine du Pin au Haras, F-61130 Exme, France \\ Courriel : aufrere@clermont.inra.fr
}

\begin{abstract}
La valeur nutritive des fourrages dépend étroitement de la digestibilité de la Matière Organique (dMO) qui est généralement mesurée in vivo sur des moutons. Au delà des valeurs moyennes figurant dans les Tables publiées par l'INRA en 2007, pour affiner l'estimation de la valeur nutritive d'un fourrage donné, il est nécessaire de pouvoir prédire la dMO de la manière la plus précise possible. Pour cela, des méthodes de prévision issues de l'analyse de la composition chimique du fourrage ou d'autres mesures réalisées en laboratoire ont été établies.
\end{abstract}

Les méthodes chimiques de prévision s'appuient sur le fait qu'il existe une liaison étroite entre la dMO et la teneur en parois indigestibles (Jarrige et Minson 1964, Baumont 2007). Comme les teneurs en parois totales et en parois indigestibles varient dans le même sens, la détermination des constituants pariétaux explique pour une large part les variations de digestibilité entre fourrages. Ainsi, des équations basées sur la teneur en Cellulose Brute (CB) ont été établies (INRA 1981, Baumont 2007) et dans certains cas leur précision est améliorée quand on associe la teneur en Matières Azotées Totales (MAT), constituant qui varie le plus avec l'âge de la plante. Le critère $\mathrm{CB}$ peut être remplacé par la teneur en ADF puisqu'il existe une relation très étroite entre ces deux paramètres. Toutefois, une prévision précise nécessite d'appliquer des équations spécifiques par espèce végétale et cycle de végétation (INRA 1981), ce qui implique une connaissance détaillée préalable du fourrage analysé.

Les méthodes biologiques basées sur la simulation in vitro de la digestion microbienne se sont largement développées (Tilley et Terry 1963, Menke et al 1979, Omed et al 2000, Adesogan 2002, Schubiger et al 2002) car elles sont corrélées de façon très étroite avec la dMO (Weiss 1994). L'inconvénient majeur porte sur l'obligation de disposer d'animaux donneurs de contenus digestifs, la nécessité de contrôler l'activité de cet inoculum et la difficulté de corriger par des standards (Jones et Theodorou 2000). Ces méthodes restent actuellement largement utilisées pour la recherche mais peu pour l'analyse en routine (Krishnamoorthy et al 2005).

Aussi un grand nombre de méthodes utilisant des enzymes du commerce avec des prétraitements différents (pepsine en milieu acide, NDF...), avec des variantes sur les températures et les durées d'incubation se sont largement développées (Broderick 1998). Les méthodes enzymatiques présentent certains avantages : leur faible coût d'analyse, la facilité de mise en œuvre puisqu'elles ne nécessitent pas d'inoculum, leur répétabilité et reproductibilité élevées. En France, Jarrige et Thivend (1969) ont utilisé une cellulase fongique, seule, pour prévoir la dMO des fourrages. Une méthode pepsine-cellulase a ensuite été développée (Andrieu 1979, Aufrère 1982) et utilisée par les laboratoires d'analyses ; elle a pu être appliquée également à la prévision de la digestibilité des aliments concentrés et composés (Aufrère et MichaletDoreau 1983). Un des avantages importants de cette méthode est qu'elle est applicable aux associations de fourrages et aux prairies permanentes.

Les équations de prévision de la dMO des fourrages utilisées actuelle- ment avec cette méthode ont été établies en 1989 sur des échantillons d'espèces pures de graminées, de légumineuses et de prairies naturelles riches en graminées (Aufrère et Demarquilly 1989). La mise à jour de ces équations par l'apport de données plus récentes couvrant une gamme plus importante de fourrages nous est apparue nécessaire pour répondre à la demande des utilisateurs. Ainsi, les nouvelles équations décrites dans ce texte reposent sur un nombre d'échantillons quatre fois plus important et permettent d'estimer la dMO sur les différentes formes d'utilisation de l'herbe, en vert, en foin ou en fourrage fermenté. Cette réactualisation ne concerne ni le maïs (Andrieu et Aufrère 1996) ni la luzerne déshydratée (Demarquilly et al 1996) pour lesquels des équations spécifiques ont été proposées.

\section{1 / La base de données et les mesures réalisées}

La banque d'échantillons que nous avons utilisée est constituée de 384 échantillons, les 85 échantillons ayant servi à établir le modèle de 1989 et 299 nouveaux échantillons. L'ensemble représente les principales catégories de fourrages verts, de fourrages fermentés (ensilages coupe directe, préfanés et mi-fanés) et de foins présents en France. Il représente également les principales espèces cultivées de graminées (ray-grass anglais, italien, hybride, dactyle, fléole et fétuque élevée), et de légumineuses (luzerne et trèfle violet) ainsi que des mélanges semés raygrass/trèfle blanc et des prairies permanentes. Ces échantillons de fourrages sont issus d'essais menés en 


\section{Détermination de la digestibilité des fourrages et des aliments concentrés par une méthode à la pepsine - cellulase}

\section{Principe}

L'échantillon subit deux attaques successives par deux enzymes diluées dans des tampons appropriés :

- un prétraitement par la pepsine pendant $24 \mathrm{~h}$ en milieu acide chlorhydrique $0,1 \mathrm{~N}$ suivi d'une hydrolyse à $80^{\circ}$ $30 \mathrm{mn}$ exactement

- un traitement par la cellulase dans un tampon acétate de sodium $0,05 \mathrm{M}, \mathrm{pH} 4,6$ pendant $24 \mathrm{~h}$.

Réactifs :

- $\mathrm{HCl} 0,1 \mathrm{~N}$

- Acétate de sodium 0,05 M pH 4,6

- Pepsine (2 g de pepsine Merck dans I litre d'HC1 0,1 N)

- Cellulase ("Onozuka R10" extraite de Trichoderma viride), $1 \mathrm{~g} / \mathrm{L}$ de tampon acétate de sodium.

\section{Mode opératoire}

Les traitements ont lieu dans des creusets de $90 \mathrm{ml}$ (hauteur $12,5 \mathrm{~cm}$, diamètre $3 \mathrm{~cm}$ ) munis d'un filtre en verre fritté de porosité 2. Peser $500 \mathrm{mg}$ d'échantillon broyé à la grille de $0,8 \mathrm{~mm}$, dans chaque creuset. Toutes les déterminations sont effectuées en triple.

Pour les aliments concentrés, il est recommandé de travailler sur des échantillons non séchés. Pour les fourrages, la température de séchage ne doit pas dépasser $80^{\circ} \mathrm{C}$.

Parallèlement déterminer la teneur en matière sèche $\left(48 \mathrm{~h}\right.$ à $\left.103^{\circ} \mathrm{C}\right)$ ainsi que la teneur en cendres $\left(5 \mathrm{~h}\right.$ à $\left.550^{\circ} \mathrm{C}\right)$ pour chaque échantillon.

Un échantillon témoin est également introduit dans chaque série pour permettre d'estimer les différences entre les séries.

1) Introduire dans chaque creuset $50 \mathrm{~mL}$ de mélange pepsine- $\mathrm{HC} 10,1 \mathrm{~N}$ à $40^{\circ} \mathrm{C}$.

Fermer avec un bouchon. Placer les creusets $24 \mathrm{~h}$ au bain-marie à $40^{\circ} \mathrm{C}$ Les creusets sont agités régulièrement 2 à 3 fois par jour.

Au bout de 24 heures :

2) Effectuer une hydrolyse acide : placer les creusets $30 \mathrm{mn}$ exactement dans un bain-marie à $80^{\circ} \mathrm{C}$.

Filtrer rapidement les creusets et rincer individuellement chaque creuset avec environ $300 \mathrm{ml}$ d'eau distillée.

3) Introduire dans chaque creuset $50 \mathrm{~mL}$ de préparation cellulasique dans le tampon acétate de $\mathrm{Na} 0,05 \mathrm{M}$, $\mathrm{pH} 4,6$. Placer les creusets $24 \mathrm{~h}$ au bain-marie à $40^{\circ} \mathrm{C}$.

Au bout de 24 heures

Filtrer chaque creuset. Rincer le résidu avec environ $300 \mathrm{~mL}$ d'eau distillée. - Sécher les creusets $48 \mathrm{~h}$ à $103^{\circ} \mathrm{C}$ et les peser. - Passer les creusets $5 \mathrm{~h}$ à $550^{\circ} \mathrm{C}$ et les peser.

\section{Calculs}

$\mathrm{E}(\mathrm{MS})$ : prise d'essai en $\mathrm{MS} ; \mathrm{E}(\mathrm{MO})$ : prise d'essai en $\mathrm{MO}$

DCS : digestibilité pepsine-cellulase de la matière sèche; DCo : digestibilité pepsine-cellulase de la matière organique

P0 : poids du creuset vide ; P1 : poids du creuset + résidu en MS ; P2 : poids du creuset + résidu cendres

$$
\begin{aligned}
& \mathrm{dCs}=100 \times[\mathrm{E}(\mathrm{MS})-(\mathrm{P} 1-\mathrm{P} 0)] / \mathrm{E}(\mathrm{MS}) \\
& \mathrm{dCo}=100 \times[\mathrm{E}(\mathrm{MO})-(\mathrm{P} 1-\mathrm{P} 2)] / \mathrm{E}(\mathrm{MO})
\end{aligned}
$$

Correction par le témoin :

[DCéchantillon x DCtémoin] / DCtémoin = DCcorrigée de l'échantillon

Normandie à l'INRA du Pin-au-Haras (fourrages verts de ray-grass anglais, de prairies permanentes, mélanges raygrass anglais/trèfle blanc, ensilages de trèfle violet) et en Auvergne à l'INRA de Theix (autres fourrages de graminées, de légumineuses).

La digestibilité in vivo (dMO) de tous ces fourrages a été mesurée sur des moutons alimentés ad libitum soit à l'INRA de Theix, soit à l'INRA du Pinau-Haras (Demarquilly et al 1995). La digestibilité pepsine-cellulase (Aufrère et Michalet-Doreau 1983) a été déterminée à l'INRA de Theix pour l'ensemble des échantillons (voir encadré). Elle a été systématiquement exprimée par rapport à la matière sèche du fourrage $(\mathrm{dCs})$ et par rapport à sa matière organique (dCo). Lorsque les fourrages sont riches en matières minérales, il est préférable de déterminer la dCo et d'utiliser les équations correspondantes. Pour les fourrages fermentés, nous avons également calculé les valeurs de dCs et de dCo corrigées pour les pertes de produits volatils lors du séchage à l'étuve.
Les équations correspondantes sont à utiliser lorsque ce coefficient de correction est déterminé (INRA 1981).

Le tableau 1 présente les valeurs moyennes de composition chimique des fourrages étudiés et la plage de variation des valeurs de dMO ainsi que les valeurs extrêmes des caractéristiques mesurées afin de connaître les limites du domaine de validité des équations proposées. Les plages de variation des fourrages de la base de données reflètent bien la variabilité 
Tableau 1. Valeurs moyennes, plages de variation de la composition chimique (Matières azotées totales, MAT, cellulose brute CB, matière minérale $M M)$, de la digestibilité pepsine-cellulase exprimée en \% MS (dCs) et \% MO (dCo) et de la digestibilité de la MO (dMO).

\begin{tabular}{|c|c|c|c|c|c|c|c|c|c|}
\hline \multirow{3}{*}{ Fourrages verts } & \multicolumn{3}{|c|}{$\begin{array}{c}\text { Graminées et prairies } \\
\text { permanentes }\end{array}$} & \multicolumn{3}{|c|}{ Légumineuses } & \multicolumn{3}{|c|}{$\begin{array}{l}\text { Ray-grass - } \\
\text { Trèfle blanc }\end{array}$} \\
\hline & \multicolumn{3}{|c|}{$N=177$} & \multicolumn{3}{|c|}{$N=32$} & \multicolumn{3}{|c|}{$N=60$} \\
\hline & moyenne & mini & maxi & moyenne & mini & maxi & moyenne & mini & $\operatorname{maxi}$ \\
\hline MAT $(\mathrm{g} / \mathrm{kg})$ & 150,3 & 69,0 & 266,0 & 187,2 & 108,0 & 253,0 & 149,3 & 86,0 & 223,0 \\
\hline CB $(\mathrm{g} / \mathrm{kg})$ & 262,9 & 186,0 & 405,0 & 267,9 & 170,0 & 347,0 & 246,2 & 185,0 & 313,0 \\
\hline MM $(\mathrm{g} / \mathrm{kg})$ & 109,6 & 55,0 & 193,0 & 122,0 & 87,0 & 150,0 & 105,0 & 79,0 & 135,0 \\
\hline $\mathrm{dCs}(\%)$ & 68,2 & 39,8 & 84,4 & 66,9 & 57,5 & 76,8 & 72,7 & 60,2 & 86,5 \\
\hline dCo $(\%)$ & 67,8 & 36,9 & 85,7 & 63,8 & 52,5 & 75,5 & 71,3 & 57,9 & 86,9 \\
\hline $\mathrm{dMO}\left(\%{ }^{\circ}\right.$ & 72,5 & 48,8 & 84,6 & 66,5 & 57,2 & 78,1 & 72,9 & 61,6 & 83,2 \\
\hline $\begin{array}{l}\text { Fourrages } \\
\text { fermentés }\end{array}$ & \multicolumn{3}{|c|}{$N=39$} & \multicolumn{3}{|c|}{$\mathrm{N}=25$} & & & \\
\hline & moyenne & mini & maxi & moyenne & $\operatorname{mini}$ & maxi & & & \\
\hline MAT $(\mathrm{g} / \mathrm{kg})$ & 129,9 & 79,0 & 203,0 & 160,7 & 108,0 & 196,0 & & & \\
\hline CB $(\mathrm{g} / \mathrm{kg})$ & 308,5 & 230,0 & 378,0 & 295,9 & 219,0 & 367,0 & & & \\
\hline $\mathrm{MM}(\mathrm{g} / \mathrm{kg})$ & 94,5 & 71,0 & 147,0 & 103,5 & 86,0 & 132,0 & & & \\
\hline dCsnc $(\%)$ & 60,9 & 47,1 & 72,6 & 64,0 & 55,2 & 72,8 & & & \\
\hline $\mathrm{dCsc}(\%)$ & 62,3 & 48,3 & 74,2 & 65,2 & 58,6 & 73,6 & & & \\
\hline dConc (\%) & 59,3 & 44,0 & 73,4 & 60,3 & 49,5 & 69,3 & & & \\
\hline $\mathrm{dCoc}(\%)$ & 60,8 & 45,2 & 75,5 & 61,7 & 49,5 & 69,3 & & & \\
\hline $\mathrm{dMO}\left(\%^{\circ}\right.$ & 68,5 & 61,4 & 74,7 & 63,4 & 57,9 & 67,4 & & & \\
\hline \multirow[t]{2}{*}{ Foins } & \multicolumn{3}{|c|}{$N=37$} & \multicolumn{3}{|c|}{$N=14$} & & & \\
\hline & moyenne & mini & maxi & moyenne & $\operatorname{mini}$ & maxi & & & \\
\hline MAT $(\mathrm{g} / \mathrm{kg})$ & 109,0 & 58,0 & 200,0 & 153,2 & 64,0 & 197,0 & & & \\
\hline CB $(\mathrm{g} / \mathrm{kg})$ & 320,2 & 203,0 & 391,0 & 321,3 & 252,0 & 380,0 & & & \\
\hline $\mathrm{MM}(\mathrm{g} / \mathrm{kg})$ & 85,3 & 38,0 & 127,0 & 99,4 & 81,0 & 115,0 & & & \\
\hline dCs (\%) & 54,7 & 38,6 & 76,2 & 60,7 & 51,8 & 69,1 & & & \\
\hline $\mathrm{dCo}(\%)$ & 52,8 & 36,9 & 75,3 & 57,4 & 48,4 & 66,1 & & & \\
\hline $\mathrm{dMO}\left(\%^{\circ}\right.$ & 61,0 & 50,2 & 72,5 & 58,3 & 54,0 & 64,7 & & & \\
\hline
\end{tabular}

présente dans les tables de la valeur alimentaire des fourrages, (INRA 1988) avec des digestibilités variant de plus de 80 pour les fourrages verts récoltés à des stades végétatifs jusqu'à 50 pour les fourrages verts et les foins récoltés aux stades les plus tardifs. La plage de variation des ensilages est plus faible, leurs stades de récolte étant moins variables. Les différences entre graminées et légumineuses présentes dans la base sont logiques, à savoir pour les légumineuses des teneurs en MAT en moyenne plus élevées et une digestibilité un peu plus faible à même teneur en cellulose brute. Les plages de variations de la composition chimique et de la digestibilité des mélanges ray-grass/ trèfle blanc ainsi que des prairies permanentes sont proches de celles des espèces cultivées de graminées.

\section{2 / Les nouvelles équations}

Pour établir les équations de prévision de la dMO nous avons procédé pour chaque mode de conservation à une analyse de covariance incluant la $\mathrm{dCs}$ ou la dCo comme variable explica- tive et l'effet de la famille végétale comme facteur fixe par la procédure GLM du logiciel SAS. Comme il n'est pas apparu de différence significative entre les graminées cultivées et les prairies permanentes, ni sur les pentes ni sur les ordonnées à l'origine des équations, nous avons regroupé ces deux catégories de fourrages dans les équations de prévision. Pour les fourrages verts nous avons donc distingué d'une part les graminées et les prairies permanentes, d'autre part les mélanges raygrass/trèfle blanc et enfin les légumineuses.

Les équations sont rapportées dans le tableau 2 et la figure 1. Le pourcentage de la variance expliqué par les équations de prévision varie de $77 \%$ pour les foins à $85 \%$ pour les fourrages verts de légumineuses. Les écarts-types de prévision sont compris entre 1,6 pour les ensilages et 2,9 pour les fourrages verts de graminées et de prairies permanentes. Pour les fourrages verts, la pente de l'équation de prévision est significativement plus élevée pour les légumineuses que pour les graminées et les prairies permanentes alors que pour les foins et les fourrages fermentés, celle-ci est commune aux graminées et aux légumineuses. De plus, les pentes des équations de prévision sont proches pour les foins et les fourrages verts de graminées alors qu'elles sont plus faibles pour les fourrages fermentés.

Comme nous l'avons observé auparavant et en accord avec les résultats de Terry et al (1978), à même digestibilité in vivo, la matière sèche solubilisée par la pepsine-cellulase est plus faible pour les fourrages verts que pour les foins, et pour les graminées que pour les légumineuses. Cela résulte du fait que la pepsine-cellulase dissout moins de constituants pariétaux que les enzymes ou les microbes du rumen et du tube digestif du ruminant. On sait qu'à même digestibilité in vivo les fourrages verts sont plus riches que les foins en constituants pariétaux et les graminées plus riches que les légumineuses. Les fourrages fermentés (ensilages) qui ont une teneur en cellulose brute plus élevée que les fourrages verts, par suite d'une disparition de constituants solubles dans le silo, sont moins dégradés que les fourrages verts par la pepsine- 
Tableau 2. Equations de prévision, coefficient de régression (R2), écart-type résiduel (ETR), de la digestibilité de la MO (dMO) à partir de la digestibilité pepsine-cellulase exprimée par rapport à la matière sèche (dCs) u de la matière organique (dCo du fourrage, et dans le cas des fourrages fermentés non corrigéé par le coefficient de correction de la matière sèche (dCsnc ou corrigée (dCsc).

\begin{tabular}{|c|c|c|c|c|}
\hline & & Equations & $\mathbf{R}^{2}$ & ETR \\
\hline \multicolumn{5}{|l|}{ Fourrages verts } \\
\hline A partir de dCs & $\begin{array}{l}\text { Graminées et prairies permanentes } \\
\text { Ray-grass - Trèfle Blanc } \\
\text { Légumineuses }\end{array}$ & $\begin{array}{l}\mathrm{dMO}=0,630 \mathrm{dCs}+29,7 \\
\mathrm{dMO}=0,630 \mathrm{dCs}+26,4 \\
\mathrm{dMO}=0,949 \mathrm{dCs}+3,00\end{array}$ & $\begin{array}{l}0,78 \\
0,86\end{array}$ & $\begin{array}{l}2,94 \\
2,21\end{array}$ \\
\hline A partir de dCo & $\begin{array}{l}\text { Graminées et prairies permanentes } \\
\text { Ray-grass - Trèfle Blanc } \\
\text { Légumineuses }\end{array}$ & $\begin{array}{l}\mathrm{dMO}=0,597 \mathrm{dCo}+32,3 \\
\mathrm{dMO}=0,597 \mathrm{dCo}+29,8 \\
\mathrm{dMO}=0,853 \mathrm{dCo}+12,0\end{array}$ & $\begin{array}{l}0,79 \\
0,85 \\
\end{array}$ & $\begin{array}{l}2,82 \\
2,28 \\
\end{array}$ \\
\hline \multicolumn{5}{|c|}{ Fourrages fermentés } \\
\hline A partir de dCs & $\begin{array}{l}\text { Graminées et prairies permanentes } \\
\text { Légumineuses } \\
\text { Graminées et prairies permanentes } \\
\text { Légumineuses }\end{array}$ & $\begin{array}{l}\mathrm{dMO}=0,459 \mathrm{dCsnc}+40,5 \\
\mathrm{dMO}=0,459 \mathrm{dCsnc}+34,0 \\
\mathrm{dMO}=0,474 \mathrm{dCsc}+38,9 \\
\mathrm{dMO}=0,474 \mathrm{dCsc}+32,5\end{array}$ & $\begin{array}{l}0,83 \\
0,84\end{array}$ & $\begin{array}{l}1,69 \\
1,66\end{array}$ \\
\hline A partir de dCo & $\begin{array}{l}\text { Graminées et prairies permanentes } \\
\text { Légumineuses } \\
\text { Graminées et prairies permanentes } \\
\text { Légumineuses }\end{array}$ & $\begin{array}{l}\mathrm{dMO}=0,432 \mathrm{dConc}+42,8 \\
\mathrm{dMO}=0,432 \mathrm{dCoc}+37,3 \\
\mathrm{dMO}=0,450 \mathrm{dCoc}+41,1 \\
\mathrm{dMO}=0,450 \mathrm{dCoc}+35,6\end{array}$ & $\begin{array}{l}0,85 \\
0,86\end{array}$ & $\begin{array}{l}1,62 \\
1,58\end{array}$ \\
\hline \multicolumn{5}{|l|}{ Foins } \\
\hline A partir de dCs & $\begin{array}{l}\text { Graminées et prairies permanentes } \\
\text { Légumineuses }\end{array}$ & $\begin{array}{l}\mathrm{dMO}=0,626 \mathrm{dCs}+26,8 \\
\mathrm{dMO}=0,626 \mathrm{dCs}+20,3\end{array}$ & 0,77 & 2,58 \\
\hline A partir de dCo & $\begin{array}{l}\text { Graminées et prairies permanentes } \\
\text { Légumineuses }\end{array}$ & $\begin{array}{l}\mathrm{dMO}=0,599 \mathrm{dCo}+29,4 \\
\mathrm{dMO}=0,599 \mathrm{dCo}+24,1\end{array}$ & 0,77 & 2,63 \\
\hline
\end{tabular}

cellulase. Cet effet est considérablement réduit pour les balles rondes enrubannées (MS > $50 \%$ ) qui se rapprochent des foins.

Les nouvelles équations permettent de prévoir pour les fourrages verts et les foins, des valeurs de dMO assez proches de celles de 1989 pour les plages de validité considérées avec toutefois une précision légèrement inférieure pour les fourrages verts de graminées, mais ces équations sont vraisemblablement plus robustes car elles intègrent un plus grand nombre de données. Afin d'étendre l'applicabilité des équations, l'espèce, le stade ainsi que le cycle de végétation n'ont pas été pris en compte dans le modèle. Ceci induit une variabilité résiduelle un peu plus élevée, comme l'ont observée

Figure 1. Liaisons entre la digestibilité de la matière organique (dMO en \%) et la digestibilité pepsine-cellulase (DCS en \% de la MS) pour les différentes catégories de fourrages verts et de fourrages conservés. Les graminées regroupent les espèces pures et les prairies permanentes. $(N=384)$.

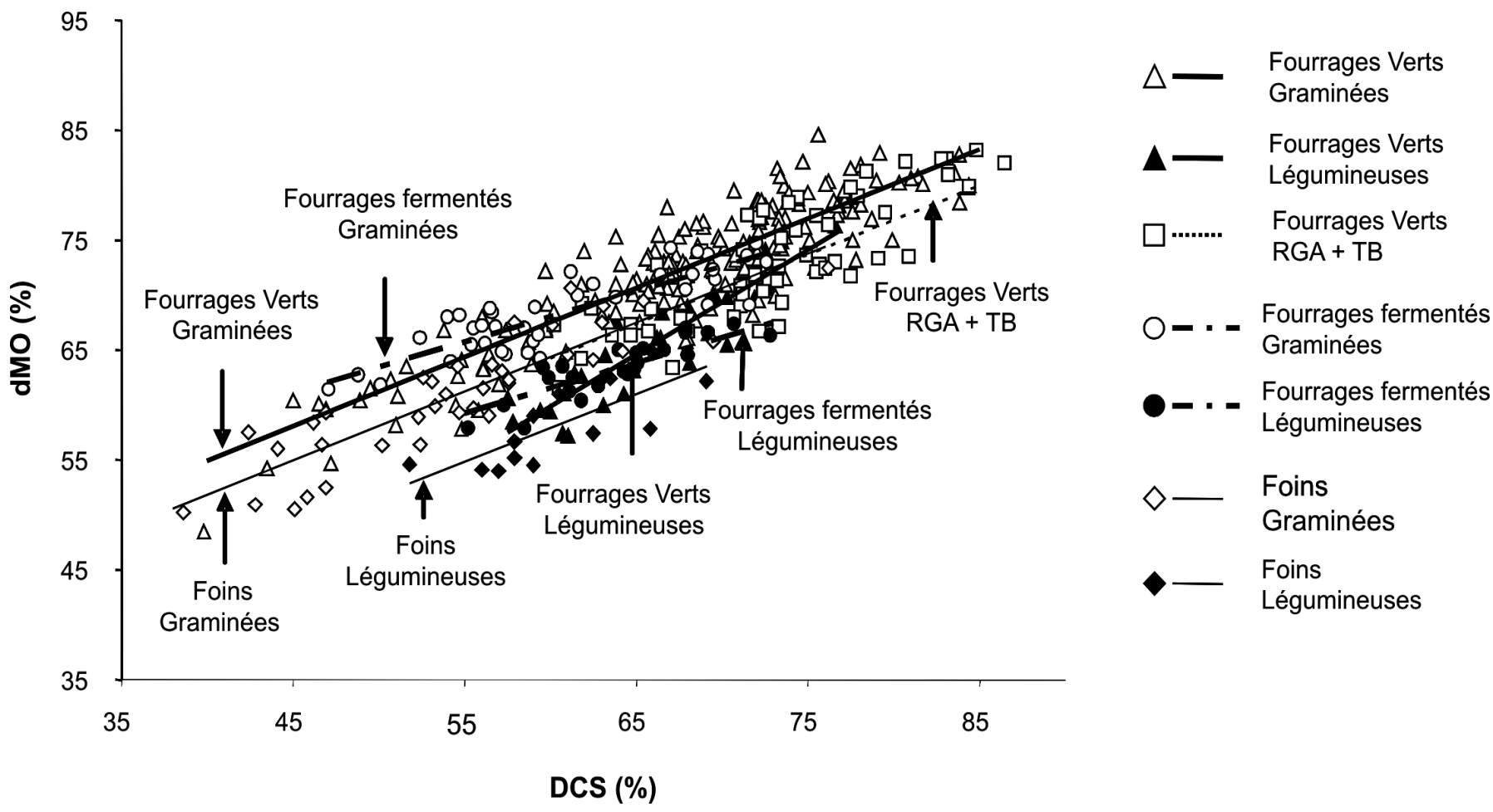


Barber et al (1990), Givens et al (1995). La précision obtenue est de l'ordre de celle observée par Givens et al (1990), mais sans conséquence sur la prévision de la valeur énergétique des fourrages. Elle est légèrement améliorée lorsque les résultats sont exprimés en matière organique en accord avec Iantcheva et al (1999).

Pour les fourrages fermentés, les équations sont précises $(\mathrm{ETR}=1,7)$ comme l'ont montré Givens et al (1993), De Boever et al (1996), Nousiainen et al (2003) et Rinne et al (2006). Les équations de prévision les plus satisfaisantes sont celles utilisant la digestibilité pepsine-cellulase corrigée par le coefficient de correction de la matière sèche lié aux pertes des produits volatils durant le séchage à l'étuve $(\mathrm{ETR}=1,6)$. Si le laboratoire ne dispose pas de ces analyses, les équations sont également calculées avec une précision un peu plus faible à partir de la digestibilité pepsine-cellulase non corrigée par ce coefficient $(\mathrm{ETR}=1,7)$. Désormais il est donc possible de prévoir la digestibilité à partir d'un échantillon de fourrage fermenté, même si l'analyse d'un échantillon prélevé à la mise en silo demeure préférable en raison de sa meilleure représentativité.

\section{3 / Validation et comparai- son avec la prévision à partir de la composition chimique}

Afin de déterminer la précision de la prévision de la dMO dans les conditions de la pratique, nous avons utilisé les nouvelles équations proposées pour les fourrages verts et les foins sur des échantillons $(n=122)$ différents de ceux utilisés pour établir les équations de prévision $(n=384)$. La dMO de ces échantillons a été mesurée sur moutons dans des lieux différents, au cours de différentes années et la dCs a été déterminée dans un même laboratoire d'analyse extérieur. De plus, cette approche a été comparée à la prévision de la dMO à partir de la composition chimique (CB, MAT) selon les équations issues du livre INRA (1981) et proposées dans le logiciel PrévAlim (Baumont et al 1999).

Les écarts moyens de prévision varient entre $-1,5$ et $+1,7$ points de digestibilité selon les catégories de fourrages (tableau 3). Ils sont comparables entre les deux méthodes, prévision par la digestibilité pepsine-cellulase et prévision par la composition chimique. En revanche, la précision de la prévision, évaluée par l'écart type de prévision, est systématiquement meilleure avec la dCs. Ainsi l'écart type de prévision varie entre 2,7 et 3,2 points de digestibilité pour la prévision à partir de la digestibilité pepsinecellulase, alors qu'il est toujours supérieur à 3,5 et peut dépasser 5 points de digestibilité pour la prévision à partir de la composition chimique (tableau 3). Moss et Givens (1990) et Givens et al (1993) avaient également obtenu une meilleure précision à partir de la digestibilité pepsine-cellulase qu'à partir de la composition chimique respectivement pour des foins et des fourrages verts.

Enfin, rappelons que la digestibilité pepsine-cellulase est sensible à la granulométrie des échantillons (Aufrère et Michalet-Doreau 1990) et à leur température de séchage. Dans une utilisation courante, il est important de respecter la granulométrie de la méthode ayant servi à établir les équations de référence (broyage à une grille de $0,8 \mathrm{~mm}$ ) et la température de séchage de $80^{\circ} \mathrm{C}$ (voir encadré). On sait qu'une température de séchage trop élevée $\left(>80^{\circ} \mathrm{C}\right)$ peut entraîner des modifications importantes du fourrage notamment par réactions de Maillard avec formation de complexes inaccessibles aux enzymes. Ces changements entraînent une diminution de la digestibilité pepsinecellulase. Le BIPEA (Bureau InterProfessionnel d'Etudes Analytiques) a pour mission d'organiser des circuits inter-laboratoires de contrôle des analyses pour permettre aux laboratoires des producteurs, des coopératives, de l'industrie et de la recherche de progresser dans la maîtrise des méthodes d'analyses. Le suivi du critère $\mathrm{dCs}$ est déjà réalisé depuis plusieurs années dans la commission «Fourrages» et le sera dorénavant dans la commission «Ensilages».

\section{Conclusion}

Ces nouvelles équations ont été intégrées dans le nouveau livre «Alimentation des Bovins Ovins et Caprins. Tables INRA 2007» et dans la version actualisée de Prévalim. Assises sur une banque d'échantillons beaucoup plus large que précédemment, elles permettent de prévoir la dMO des fourrages issus de graminées et de légumineuses cultivées avec une précision satisfaisante et meilleure que celle obtenue à partir de la composition chimique. Les équations proposées pour les graminées peuvent également être utilisées pour des prairies permanentes riches en graminées. Des études sont actuellement en cours afin d'étendre son application aux prairies multi espèces ou à flore complexe riches en dicotylédones. La méthode peut dorénavant être utilisée pour prévoir la dMO à partir d'un échantillon de fourrage fermenté. Des équations

Tableau 3. Moyenne des écarts entre la dMO mesurée et estimée pour des échantillons n'ayant pas été utilisés pour construire le modèle.

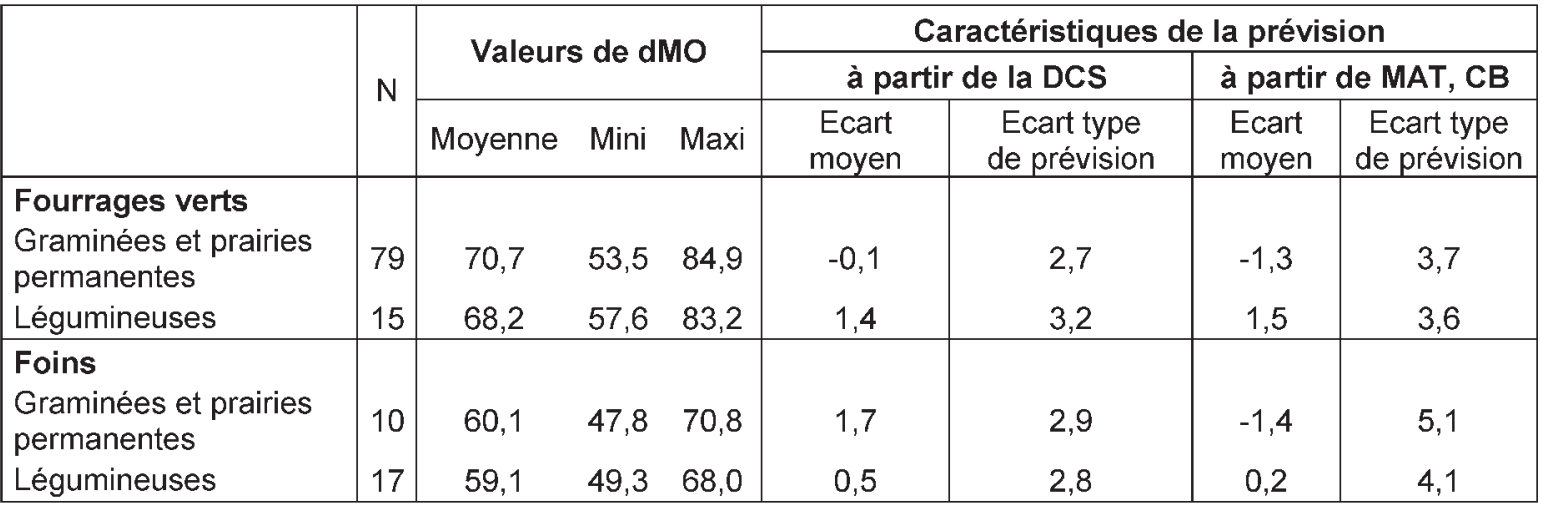


spécifiques ayant été également établies pour le maïs fourrage et pour la luzerne déshydratée, la même méthode pepsine-cellulase peut désormais être utilisée pour prévoir la digestibilité et donc la valeur énergétique des principaux fourrages utilisés dans l'alimentation des ruminants.
Dans la pratique, les équations proposées seront intégrées au logiciel Prévalim. L'utilisateur qui veut prédire la valeur alimentaire de son fourrage peut faire cette analyse, couplée à un dosage de MAT. Son coût moyen est de 30 euros HT dans les laboratoires départementaux

\section{Remerciements}

Remerciements à Mmes D. Berthon, $\mathrm{M}$. Dudilieu, D. Graviou, M. Jailler et J. Jamot pour les analyses de digestibilité pepsinecellulase, et au laboratoire du CILAL pour les analyses des échantillons de validation.

\section{Références}

Adesogan A.T., 2002. What are feeds worth? A critical evaluation of selected nutritive value methods. Proc. 13th Ann. Florida Ruminant Nutrition Symp., 33-47.

Andrieu J.P., 1979. Utilisation d'enzymes cellulolytiques pour prévoir la valeur nutritive des aliments pour les ruminants. Mémoire de D.E.A, Université de Clermont II. UER de Sciences Exactes et Naturelles. Laboratoire de Physiologie Animale Endocrinologie et Nutrition, 46p.

Andrieu J., Aufrère J., 1996. Prévision à partir de différentes méthodes (physique, chimique et biologique) de la digestibilité et de la valeur énergétique de la plante de maïs à l'état frais. In : Colloque Maïs Ensilage. 17-18 septembre 1996, AGPM (Ed.), Nantes, France, 61-69.

Aufrère J., 1982. Etude de la prévision de la digestibilité des fourrages par une méthode enzymatique. Ann. Zootech., 31, 11-30.

Aufrère J., Demarquilly C., 1989. Predicting organic matter digestibility of forage by two pepsin-cellulase methods. XVI Int. Grassl. Cong., Nice, France, 2, 877-878.

Aufrère J., Michalet-Doreau B., 1983. In vivo digestibility and prediction of digestibility of some by-products. Feeding value of by-products and their use by beef cattle. EEC Seminar, 26-29 september 1983, Melle-Gontrode, Belgique, 25-33.

Aufrère J., Michalet-Doreau B., 1990 Nouvelles méthodes d'estimation de la valeur alimentaire des fourrages. II. Méthodes enzymatiques. Fourrages, 122, 203-217.

Barber G.D., Givens D.I., Kridis M.S., Offer N.W., Murray I., 1990. Prediction of the organic matter digestibility of grass silage. Anim. Fd Sci. Technol., 28, 115-128.

Baumont R., Champciaux P., Agabriel J., Andrieu J., Aufrère J., Michalet-Doreau B., Demarquilly. C., 1999. Une démarche intégrée pour prévoir la valeur des aliments pour les ruminants : PrévAlim pour INRAtion. INRA Prod. Anim., 12, 183-194.

Baumont R., Dulphy J.P., Sauvant D., Meschy F., Aufrère J., Peyraud J.L., 2007. Valeur alimentaire des fourrages et des matières premières : tables et prévision. In : Alimentation des bovins, ovins et caprins. Besoins des animaux. Valeurs des Aliments. Tables INRA 2007. Editions Quæ, Versailles, France, 149-179.

Broderick G.A., 1998. Can cell-free enzymes replace rumen micro-organisms to model energy and protein supply? In vitro techniques for measuring nutrient supply to ruminants. E.R. Deaville, E. Owen, A.T. Adegosan, C. Rymer, J.A. Huntington, T.L.J. Lawrence (Eds), Occasional Publication, 22, 99-114.

De Boever L., Cottyn B.G., De Brabander D.L., Vanacker J.M., Boucqué Ch.V., 1996 Prediction of the feeding value of grass silages by chemical parameters, in vitro digestibility and near-infrared reflectance spectroscopy. Anim. Fd Sci. Technol., 60, 103-115.

Demarquilly C., Chenost M., Giger S., 1995. Pertes fécales et digestibilité des aliments et des rations. In : Nutrition des ruminants domestiques. Jarrige R., Ruckebusch Y., Demarquilly C., Farce M.H., Journet M. (Eds), INRA Editions, Paris, France, 501-648.

Demarquilly C., Andrieu J., Aufrère J., Graviou D., 1996. Valeur énergétique des luzernes. In : Attualita e prospetive della foraggicoltura da proto et da parcolo, Lodi, Italie, 22-24

Givens D I., Everington J.M., Adamson A.H., 1990. The nutritive value of spring-grown herbage produced on farms throughout England and Wales over 4 years. II. The prediction of apparent digestibility in vivo from various laboratory measurements. Anim Fd Sci Technol., 27, 173-184

Givens D.I., Moss A.R., Adamson A.H., 1993. Influence of growth stage and season on the energy value of fresh herbage. 2. Relationship between digestibility and metabolisable energy content and various laboratory measurements. Grass Forage Sci., 48, 175-180.

Givens D.I., Cottyn B.G., Dewey P.J.S., Steg A., 1995. A comparison of the neutral detergentcellulase method with other laboratory methods for predicting the digestibility in vivo of maize silages from three European Countries. Anim.Fd Sci. Technol., 54, 55-64.

Iantcheva N., Steingass H., Todorov N., Pavlov D., 1999. A comparison of in vitro rumen fluid and enzymatic methods to predict digestibility and energy value of grass and alfalfa hay. Anim. Fd Sci.Technol., 81, 333-344.

INRA Institut National de la Recherche Agronomique, 1981. Prévision de la valeur nutritive des aliments des ruminants. Demarquilly C. (Ed) INRA, Paris, France, 580p.

INRA Institut National de la Recherche Agronomique, 1988. Alimentation des bovins, ovins et caprins. Jarrige R. (Ed), INRA Editions, Paris, France, 471p.

INRA Institut National de la Recherche Agronomique, 2007. Alimentation des bovins, ovins et caprins. Besoins des animaux. Valeurs des aliments. Tables INRA 2007. Editions Quæ, Paris, France, 307p

Jarrige R., Minson D.J., 1964. Digestibilite des constituants du ray-grass anglais S24 et du dactyle S37, plus spécialement des constituants glucidiques. Ann. Zootech., 13, 117-153.

Jarrige R., Thivend P, 1969. Action d'une cellulase fongique sur les membranes et son intérêt pour prévoir la digestibilité des plantes fourragères. Ann. Biol. Anim. Bioch. Biophys., 9, 171-190.
Jones D.I.H., Theodorou M.K., 2000. Enzyme techniques for estimating digestibility. In Forage Evaluation in ruminant nutrition. D.I. Givens, E. Owen, R.F.E. Axford, H.M. Omed (Eds) CABI Publishing, Wallingford, UK, 155173

Krishnamoorthy U., Rymer C., Robinson P.H., 2005. The in vitro gas production technique: Limitations and opportunities. Anim. Fd Sci. Technol., 123-124, 1-578.

Menke K.H., Raab L., Salewski A., Steingass H., Fritz D., Schneider W., 1979. The estimation of the digestibility and metabolizable energy content of ruminant feedingstuffs from the gas production when they are incubated with rumen liquor in vitro. J. Agric. Sci., 93, 217-222.

Moss A.R., Givens D.I., 1990. Chemical composition and in vitro digestion to predict digestibility of field-cured and barn-cured grass hays. Anim. Fd Sci. Technol., 31, 125-138.

Nousianen J., Rinne M., Hellämäki M., Huhtanen P., 2003. Prediction of the digestibility of the primary growth of grass silages harvested at different stages of maturity from chemical composition and pepsin-cellulase solubility. ion technique: Limitations and opportunities. Anim. Fd Sci. Technol., 103, 97-111.

Omed H.M., Lovett D.K., Axford R.F.E., 2000. Faeces as a source of microbial enzymes for estimating digestibility. In: Forage Evaluation in ruminant nutrition. Givens D.I., Owren E., Axford R.F.E., Omed H.M.(Eds) CABI Publishing, Wallingford, UK, 135-154.

Rinne M., Olt A., Nousiainen J., Seppälaä A., Tuori M., Paul C., Fraser M.D., Huhtanen P., 2006. Prediction of legume silage digestibility from various laboratory methods. Grass and Forage Sci., 61, 354-362.

Schubiger F.X., Lehmann J., Daccord R., Arrigo Y., Jeangros B., Scheovic J., 2002. Comparison of laboratory methods for determining the digestibility of forage plants. Rev. Suisse Agric., 34, 1, 13-16.

Terry R.A., Mondell D.C., Osbourn D.F., 1978. Comparison of two in vitro procedures using liquor-pepsin or pepsin-cellulase for prediction of forage digestibility. J. Br. Grassl. Soc., $33,13-18$.

Tilley J.M.A., Terry R.A.,1963. A two stage technique for in vitro digestion of forage crops. J. Br. Grassl. Soc., 18, 104-111.

Weiss W.P., 1994. Estimation of digestibility of forages by laboratory methods. In: Forage quality Evaluation and Utilization. Fahey G.C., Collins Jr M., Mertens D.R., Moser L.E. (Eds), American Society of Agronomy, Crop Science Society of America And Soil Science Society of America, Madison, USA, 644-680. 


\section{Résumé}

La digestibilité pepsine-cellulase est largement utilisée pour prévoir la digestibilité de la matière organique (dMO) des fourrages. Les équations de prévision utilisées jusqu'à présent avaient été établies en 1989 sur 85 échantillons d'espèces pures de graminées, de légumineuses, et sur des prairies permanentes riches en graminées, récoltées en vert ou en foin. Certaines espèces fourragères comme le trèfle violet, les mélanges ray-grass/trèfle blanc, et les fourrages récoltés en ensilage n'avaient pas été inclus dans cette équation. Pour conforter et élargir les équations de prévision, nous avons analysé la relation entre la dMO et la digestibilité pepsine-cellulase d'une banque de données constituée de 384 échantillons dont la digestibilité in vivo a été mesurée sur moutons à l'INRA de Theix en Auvergne ou à l'INRA du Pin-au-Haras en Normandie et dont la digestibilité pepsine-cellulase a été déterminée à l'INRA de Theix. Cette banque de données comporte 269 échantillons de fourrages verts (121 de graminées, 60 de mélanges ray-grass/trèfle blanc, 32 de légumineuses et 56 de prairies permanentes), 64 échantillons de fourrages fermentés (39 de graminées, 25 de légumineuses) et 51 échantillons de foins (37 de graminées et 14 légumineuses). Sur l'ensemble des données $(n=384)$, la plage de variation de la dMO est de 48,5 à 84,6 et celle de la digestibilité pepsine-cellulase de 38,6 à 86,5. L'analyse de cette base de données nous a permis de conforter les équations de prévision proposées pour les fourrages verts et les foins (écarts-types résiduels compris en 2,2 et 2,9 points de digestibilité), de préciser les différences de pente dans les équations de prévision entre les fourrages de graminées et de légumineuses, de proposer une équation pour les fourrages verts issus d'association ray-grass/trèfle blanc et des équations pour les fourrages fermentés (ensilages et balles rondes) avec une précision inférieure à 2 points de digestibilité. Une étude de validation a été réalisée à partir de 122 échantillons extérieurs à ceux utilisés pour établir les équations de prévision et dont la composition chimique et la digestibilité pepsine-cellulase ont été déterminées dans un laboratoire d'analyse extérieur à l'INRA. Les écarts-types de prévision de la dMO à partir de la digestibilité pepsine-cellulase varient de 2,7 et 3,2 points de digestibilité selon la catégorie de fourrages considérée. Ils sont inférieurs à ceux obtenus pour la prévision à partir de la composition chimique (MAT, CB) qui varie entre 3,5 et 5,1 points de digestibilité.

\section{Abstract}

\section{Laboratory prediction of forage digestibility by the pepsin-cellulase method. The renewed equations.}

Pepsin-cellulase digestibility determination is widely used to predict forage organic matter digestibility (OMd). Prediction equations were established in 1989 on 85 fresh forage or hay samples of pure species of grasses and legumes and on permanent grasslands rich in grasses. Some species like red clover, perennial ryegrass/white clover, as well as fermented forages (silages, wrapped big bales) were not included in this model. To extend and strengthen the prediction equations, we analysed the relation between the OMd and the pepsin-cellulase digestibility from a database containing 384 measurements of OMd performed on sheep at INRA Theix in Auvergne or at INRA Pin-auHaras in Normandy. The determinations of pepsin-cellulase digestibility were all performed at INRA Theix. The database contains 269 measurements on fresh forages (121 grasses, 60 ryegrass - white clover mixtures, 32 legumes and 56 permanent grasslands), 64 measurements on fermented forages (39 grasses and 25 legumes) and 51 measurements on hays (37 grasses and 14 legumes)., OMd varied from 48.5 to 84.6 and pepsin-cellulase digestibility from 38.6 to 86.5, determined from the whole dataset. The analysis of the database allowed us to propose renewed prediction equations for fresh forages and hay with residual standard deviations between 2.2 and 2.9 points of digestibility, and to specify the differences in slope between grasses and legumes. A specific equation was proposed for rye-grass / white clover mixtures. New equations were also proposed to predict OMd of fermented forages with a residual standard deviation lower than 2 points of digestibility. A validation study was conducted on 122 external samples for which pepsin-cellulase was determined in a commercial laboratory. The standard error of prediction varied between 2.7 and 3.2 points of digestibility for the different forage types. It was lower than the standard error of prediction obtained from the chemical composition (crude fibre and crude protein) that varied between 3.5 and 5.1 points of digestibility.

AUFRÈRE J., BAUMONT R., DELABY L., PECCATE J.-R., ANDRIEU J., ANDRIEU J.-P., DULPHY J.-P., 2007. Prévision de la digestibilité des fourrages par la méthode pepsine-cellulase. Le point sur les équations proposées. INRA Prod. Anim., 20, 129-136. 
